LBNL-62173

\title{
Study of the OCS6 Lattice Using Frequency Maps
}

\author{
Ina Reichel \\ Lawrence Berkeley National Laboratory \\ 2 January 2007
}

\begin{abstract}
Frequency maps are employed to study the baseline damping ring lattice. The study is aimed at understanding the reduced dynamic aperture in the lattice with four short straight sections compared to the one with eight short straight sections. Measures to increase the dynamic aperture based on results of this study are suggested.
\end{abstract}

\section{Frequency maps}

Frequency maps have been used for some years to study dynamic aperture and related issues in particle accelerators [1]. They can help in the understanding of loss mechanisms and sources of dynamic aperture restrictions. Below is a short description how frequency maps are calculated:

A grid of particles is generated at an arbitrary starting point in the ring. All angles are zero and usually the energy deviations and longitudinal positions inside the bunch are zero as well. The horizontal amplitudes range from zero to a value corresponding to the desired dynamic aperture. The grid is not evenly spaced but the amplitude of the $n^{\text {th }}$ particle in one plane is given by $A_{n}=A_{\max } \sqrt{n / N}$ where $A_{\max }$ is the maximum amplitude and $N$ is the number of grid points in that plane. This avoids tracking many particles at low amplitudes 
where the results are not of interest while still having a useful number of points at large amplitude and keeping the computing time reasonable. For most frequency maps in this study a grid of 40 by 40 particles (which takes about 7 hours to compute) has been used except for the scans, where a 10 by 10 grid was used.

The largest amplitudes $\left(A_{\max }\right)$ used in this study correspond to values slightly smaller than the required acceptance. The horizontal dispersion at the launch point is not exactly zero but small enough to be negligible compared to $A_{\max }$ even for $\frac{\Delta p}{p}= \pm 1 \%$.

This grid of particles is then tracked for a number of turns (in this study, usually 1024 turns were used except for the scans where 256 turns were used). Tracking is done without synchrotron radiation or accelerating voltages. The amplitudes of all particles are recorded each turn. These data are then split in two: One set for the first half of turns and one for the second half. Each set is then used to calculate the tune of each particle. The two tunes for each particle are then compared and the difference, called the tune diffusion rate, is saved. The lower the diffusion rate, the more stable the particle's trajectory.

One can then plot the tune diffusion rate versus the initial amplitude or versus the tune value for the first set. Usually both ways of plotting the data are used. For the scans, all diffusion rates are summed up over all particles and only the sum is used. This is a way to scan some parameters without comparing dozens of frequency maps. The sum of the diffusion rates is not a perfect measure of the 'quality' of a frequency map, as the grid points are not equally spaced (so high diffusion rates at low amplitude do not contribute much) and it does not weigh diffusion rates or particle losses with the amplitude at which they occur (so a frequency map can have a low total diffusion rate even if some particle losses occur at very low amplitude if the rest of the frequency map has low diffusion rates). Still it is a useful tool to get an idea which area of the considered parameter space may warrant further study.

All frequency maps in this study were calculated without machine errors like alignment, strength or multipole errors as this was beyond the scope of this study. Those errors are likely to decrease the dynamic aperture, therefore they should be included in future studies. 


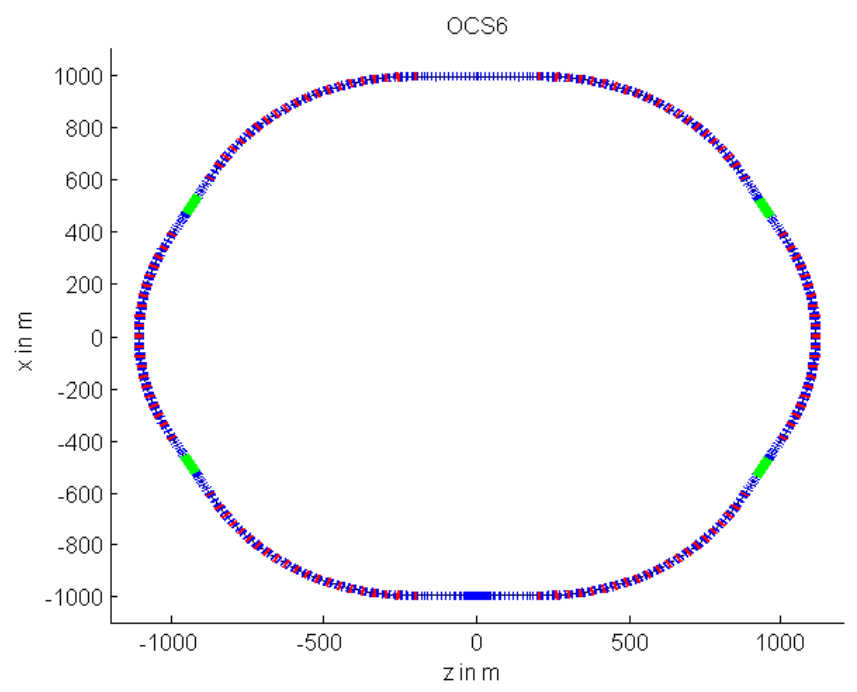

Figure 1: The OCS6 lattice.

\section{The ocs lattice}

\subsection{Description of the lattice}

The OCS6 lattice is the baseline version for the ILC Damping Rings. The lattice has four short and two long straight sections. The short straight sections contain the wiggler magnets and rf cavities. One or both long straight sections can be used for injection and extraction depending on the overall layout. A layout plot of the lattice is shown in Fig. 1.

Earlier versions of the lattice had eight short straight sections instead of four. The number of short sections was reduced to minimize the number of required access shafts in order to save costs. Upon reducing the number of straight sections it was noted that the dynamic aperture with fewer straight sections was significantly reduced. In order to identify the source of this reduction, frequency maps of the OCS6 lattice are compared to OCS5, the previous version of the lattice which still had eight short straight sections.

The OCS5 lattice has eight short (wiggler and rf) and two long (injection and extraction) straight sections. A layout plot of the lattice is shown in Fig. 2 on the next page.

The arc cells in the OCS5 and OCS6 lattices are identical. The straight sections are somewhat different and the arc cells and straights are of course laid out in 


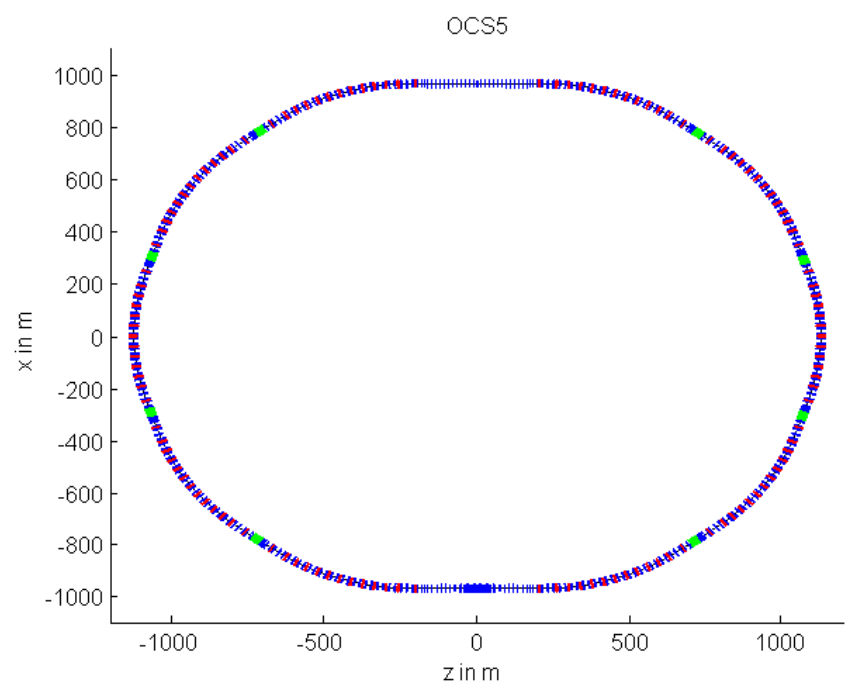

Figure 2: The OCS5 lattice.

a different way. But overall the lattices are not very different from each other making the reduction in dynamic aperture rather puzzling.

\subsection{On-momentum frequency maps}

Figure 3 on the following page shows the tune footprints for the OCS5 (Fig. 3(b)) and OCS6 (Fig. 3(a)) lattices. The nominal tunes of the lattices differ, but the different tunes are not the source of the reduced dynamic aperture (see section 3 ). The $\beta$-functions at the launch point are the same for both lattices within $0.1 \%$, so the same grid was used in both cases. The footprint is significantly smaller for the OCS5 lattice indicating a smaller detuning with amplitude. In the OCS6 lattice the cross detuning with amplitude (vertical detuning with horizontal amplitude) is particularly large. As the footprint is larger, it is more likely to cross harmful resonances.

Figure 4 on the next page shows the frequency maps for both lattices. Although some resonance lines are clearly visible for the OCS5 lattice (Fig. 4(b)), particularly the horizontal quarter-integer, the frequency map looks fine. The footprint is reasonably compact, the diffusion rates are low for most of the points and there are very few points with high diffusion rates.

For the OCS6 lattice (Fig. 4(a)), some resonance lines are visible as well, par- 


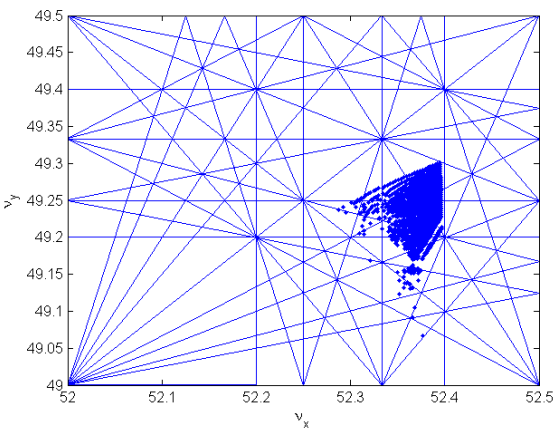

(a) OCS6

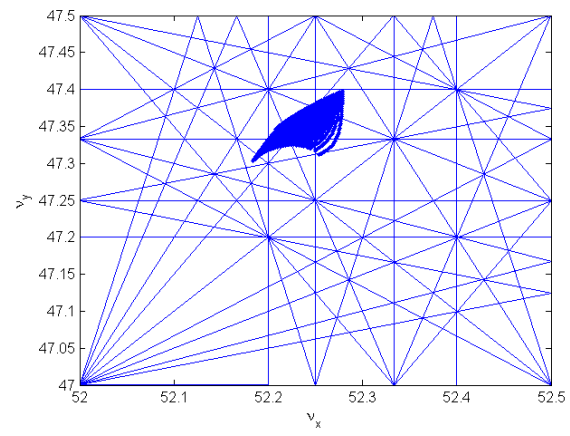

(b) OCS5

Figure 3: Tune footprints for ideal lattices. Resonances up to fifth order are shown.

ticularly the vertical quarter-integer and the horizontal third-integer. However the footprint is significantly larger than for OCS5 and it is frayed at the lower left side. Although a large number of particles have a small tune diffusion rate, some particles at larger amplitudes have high tune diffusion rates.

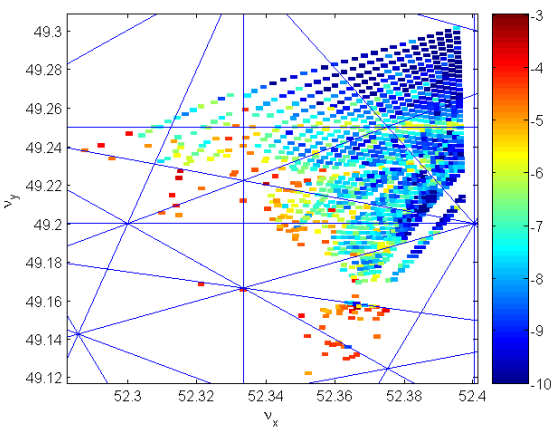

(a) OCS6

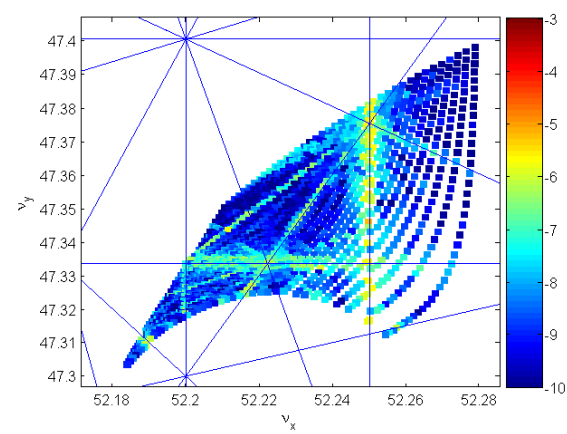

(b) OCS5

Figure 4: Frequency maps for ideal lattices. Resonances up to fifth order are shown.

Figure 5 on the following page shows the same data as Fig. 4 but plotted versus initial amplitude instead of tune. For the OcS6 lattice one can see that particles at large vertical amplitude are lost. As the largest tracked amplitude is still slightly smaller than the required aperture, this is not acceptable. Large diffusion rates occur at large vertical amplitudes even for small horizontal amplitudes. The area up to $A_{x}=30 \mathrm{~mm}$ and $A_{y}=20 \mathrm{~mm}$ is very stable. The yellow arc of higher diffusion rates at that amplitude is caused by the vertical fourth 
order resonance (see Fig. 4(a) on the previous page).

The OCS5 lattice, on the other hand, has a sufficient dynamic aperture and, except for some visible resonances, particles at large amplitude still have reasonably small diffusion rates. The only resonance showing up as larger than ideal diffusion rates is the vertical quarter-integer. This resonance is crossed at an amplitude of slightly larger than $40 \mathrm{~mm}$ in the horizontal plane and about $15 \mathrm{~mm}$ in the vertical plane.

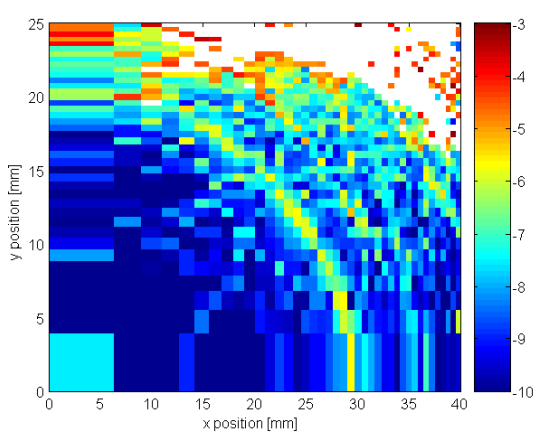

(a) OCS6

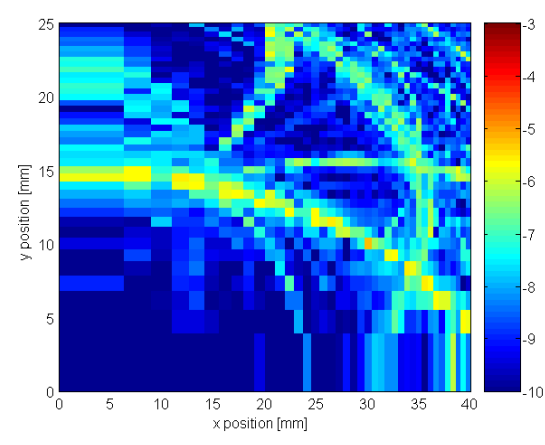

(b) OCS5

Figure 5: Tune diffusion versus amplitude for the ideal lattices. The $\beta$-functions at the launch point are the same for both lattices within $0.1 \%$.

Whereas the OCS5 lattice shows no dynamics problems in the required aperture range the OCS6 lattice clearly suffers from large detuning with amplitude, strong resonances and resulting loss of particles. The dynamic aperture even on-energy is marginal and even stable particles exhibit large tune diffusion rates, which was not observed in the ocs5 lattice.

\subsection{Off-momentum frequency maps}

As the injected positron beam has a large energy spread ( $1 \%$ full width) with a fairly flat distribution, it is important, that the dynamic aperture is also sufficient for off-momentum particles. As the tracking is done at a chromaticity of $\xi_{x}=\xi_{y}=1$, the tune will be different for the off-momentum particles.

Figure 6 on the following page shows the tune footprints for different momentum deviations for the OCS6 lattice. One can clearly see that the footprint moves around in tune space due to the chromaticity. It looks fairly compact for positive energy deviations but large and frayed out for negative ones. 


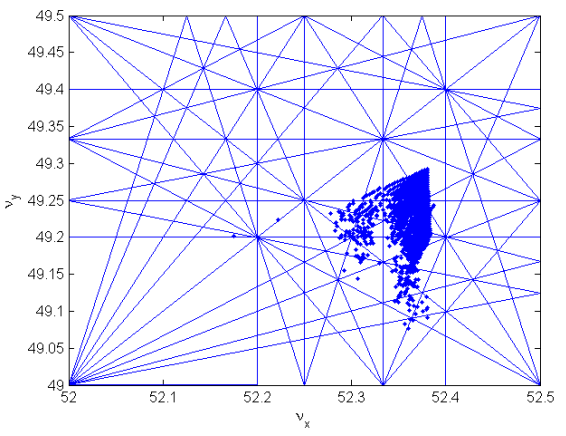

(a) $\frac{\Delta p}{p}=-0.5 \%$

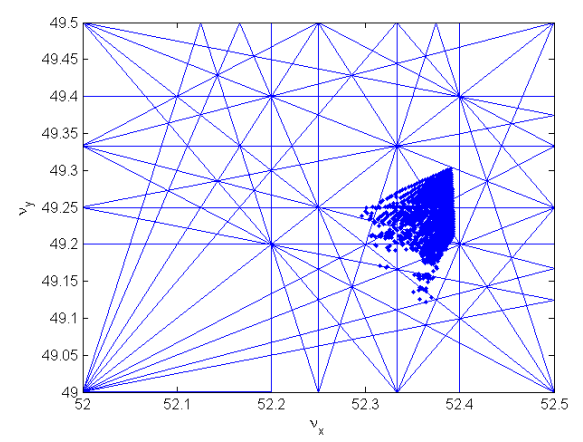

(c) $\frac{\Delta p}{p}=+0.5 \%$

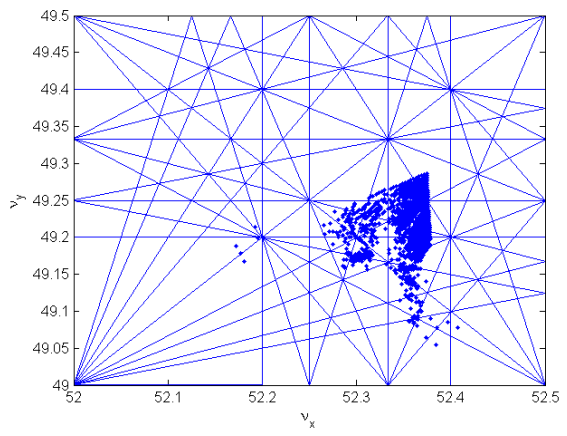

(b) $\frac{\Delta p}{p}=-1.0 \%$

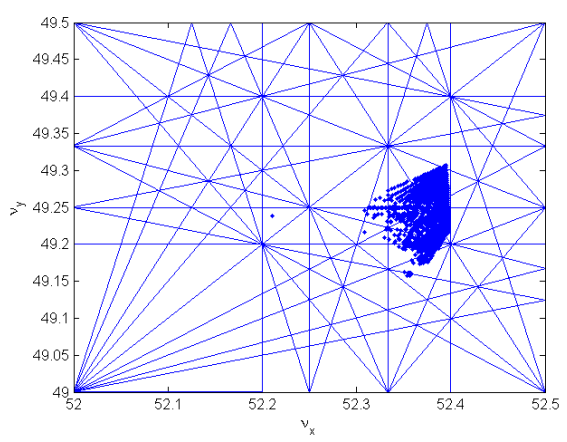

(d) $\frac{\Delta p}{p}=+1.0 \%$

Figure 6: Off-momentum footprints for the OCS6 lattice.

Figure 7 on the next page shows the off-momentum frequency maps for the nominal case $\left(\xi_{x}=\xi_{y}=1\right)$. One can clearly see the problems for negative energy deviations caused by pushing the footprint over the horizontal third integer resonance. Overall, the diffusion rates are significantly higher than in the on-momentum case (compare to Fig. 4(a) on page 5).

Figure 8 on page 9 shows the diffusion rates plotted versus amplitude. The dynamic aperture is even smaller than for the on-momentum which case. The area with low tune diffusion rates is now really small, but most resonance lines showing up at lower amplitudes do not increase the diffusion rate too much. Particles at large vertical amplitudes, even at small and moderate horizontal amplitudes, suffer from large tune diffusion rates and a significant fraction of them are lost.

The dynamic aperture of the ocs6 lattice is already marginal on-energy. For 


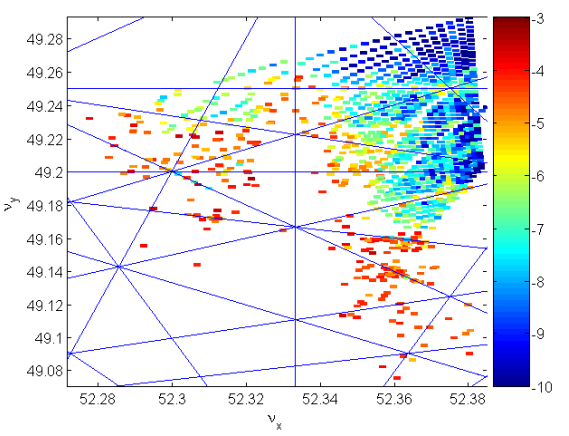

(a) $\frac{\Delta p}{p}=-0.5 \%$

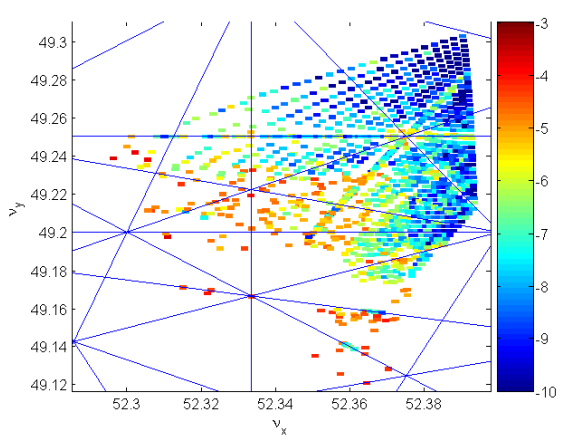

(c) $\frac{\Delta p}{p}=+0.5 \%$

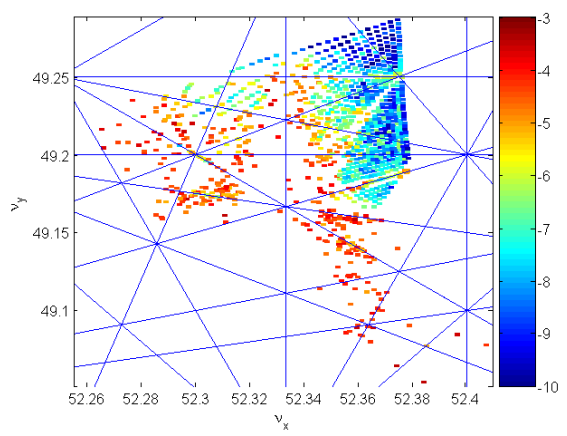

(b) $\frac{\Delta p}{p}=-1.0 \%$

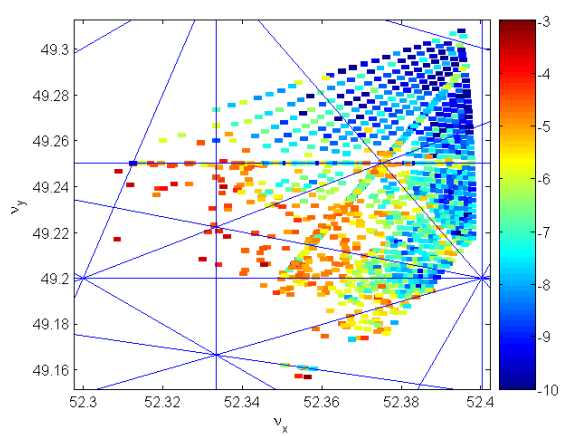

(d) $\frac{\Delta p}{p}=+1.0 \%$

Figure 7: Off-momentum frequency maps for the Ocs6 lattice.

particles with momentum deviation, it is clearly reduced further, for negative momentum deviation more so than for positive. The problems are mainly in the vertical plane where large horizontal detuning with vertical amplitude pushes particles over the horizontal third-order resonance. In addition to losing many particles, surviving particles exhibit large detuning with amplitude.

\subsection{Varying the chromaticity of the ocs6 lattice}

Most studies presented here were done with chromaticities of $\xi_{x}=\xi_{y}=1$. As it is likely that multi-bunch instabilities will limit the performance of the damping rings, it might be necessary to run at a higher chromaticity. 


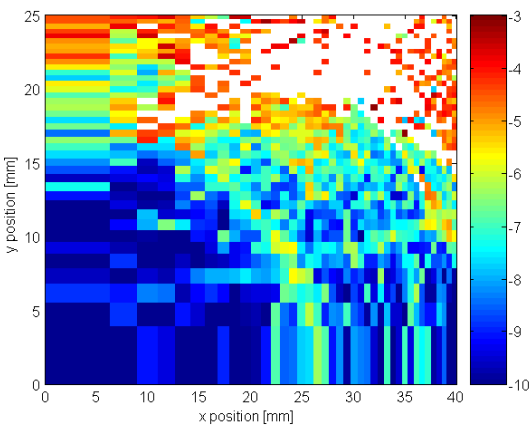

(a) $\frac{\Delta p}{p}=-0.5 \%$

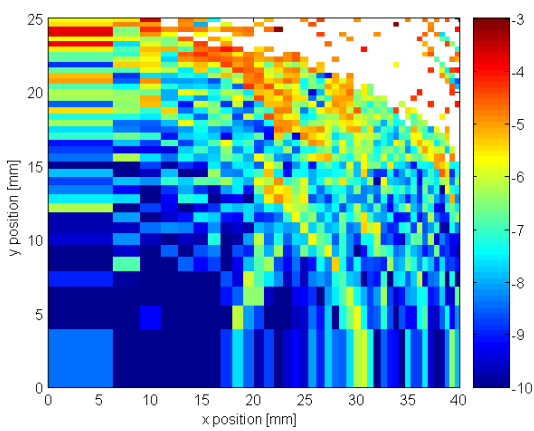

(c) $\frac{\Delta p}{p}=+0.5 \%$

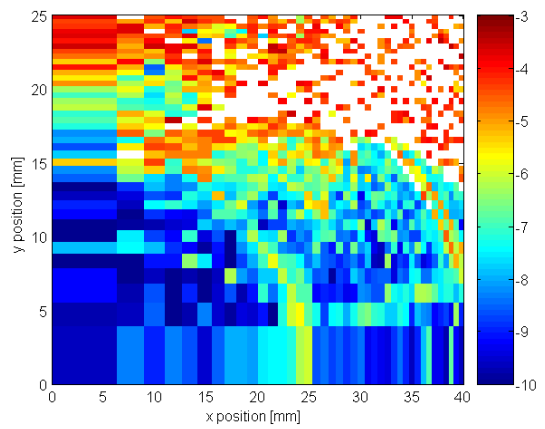

(b) $\frac{\Delta p}{p}=-1.0 \%$

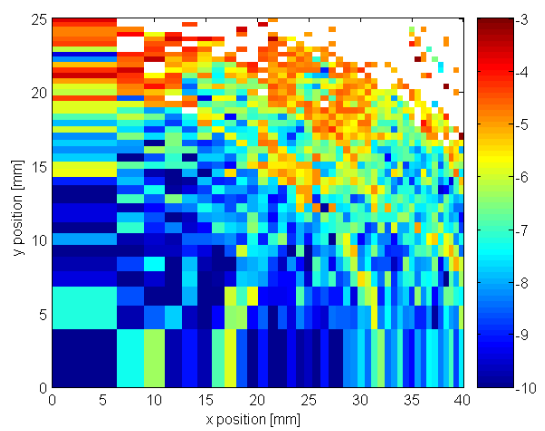

(d) $\frac{\Delta p}{p}=+1.0 \%$

Figure 8: Off-momentum tune diffusion versus amplitude for the ocs6 lattice.

\subsubsection{On-momentum}

Figures 9, 10 and 11 show the results for $\xi_{x}=\xi_{y}=3$ and the result for the nominal case $\left(\xi_{x}=\xi_{y}=1\right)$ for comparison.

Figure 9 (b) on the following page shows the tune footprint for chromaticities of $\xi_{x}=\xi_{y}=3$. One can see that the footprint is more frayed than the one for smaller chromaticity (nominal case, $\xi_{x}=\xi_{y}=1$ )

The frequency map (Fig. 10(b) on the next page) looks slightly worse, but the dynamic aperture is not decreased by much. However, one has to keep in mind that this is the on-momentum case, so the effect of the stronger sextupoles should be small.

Figure 11 on page 11 shows that the dynamic aperture is decreased by higher chromaticity, especially in the vertical plane. Also, the first clearly visible resonance (vertical fourth-order resonance) occurs at a lower amplitude. 


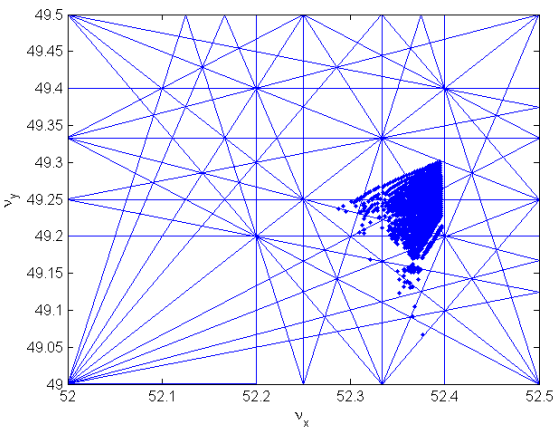

(a) $\xi_{x}=\xi_{y}=1$

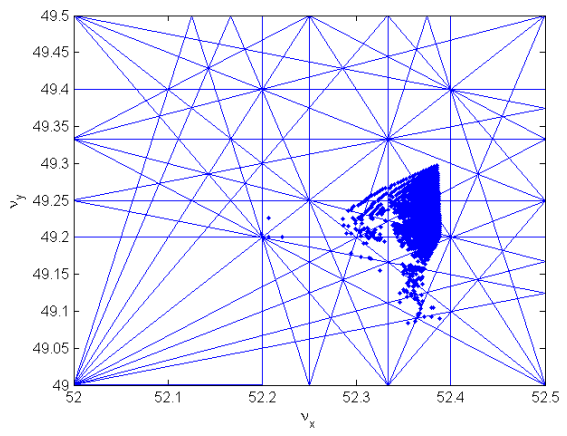

(b) $\xi_{x}=\xi_{y}=3$

Figure 9: Tune footprint for the OCS6 lattice for different chromaticities. Resonances up to fifth order are shown.

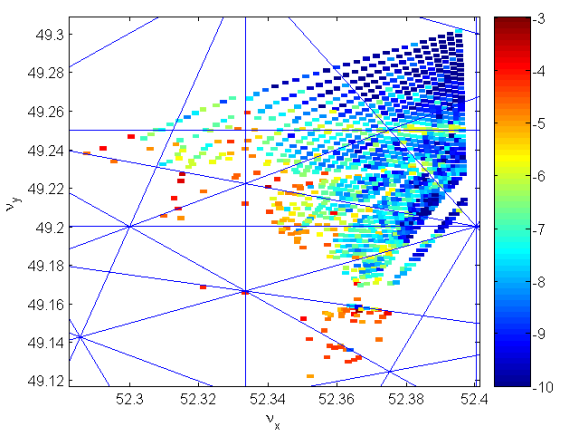

(a) $\xi_{x}=\xi_{y}=1$

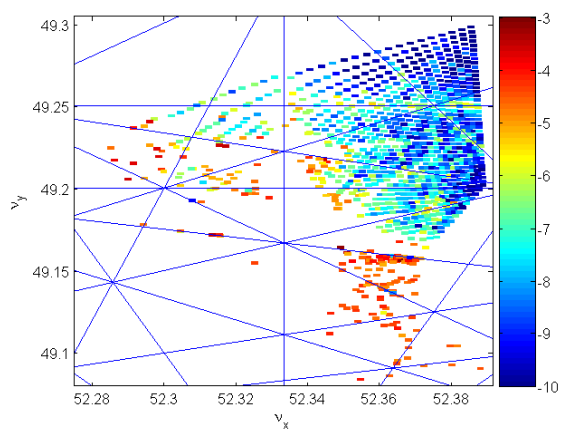

(b) $\xi_{x}=\xi_{y}=3$

Figure 10: Frequency maps for the OCS6 lattice for different chromaticities. Resonances up to fifth order are shown.

Even in the on-momentum case, where the influence of the stronger sextupole magnets should be weak, a slight reduction in dynamic aperture for a modest increase in chromaticity has been observed, making it desirable to avoid large chromaticities.

\subsubsection{Off-momentum}

Figure 12 on page 12 shows the footprints for different momentum deviations for $\xi_{x}=\xi_{y}=3$. One can see that the footprint moves around more than for the 


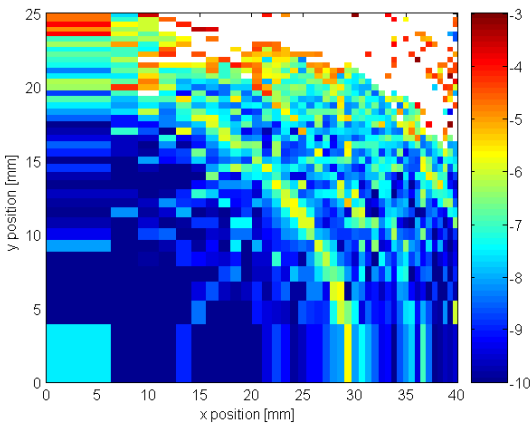

(a) $\xi_{x}=\xi_{y}=1$

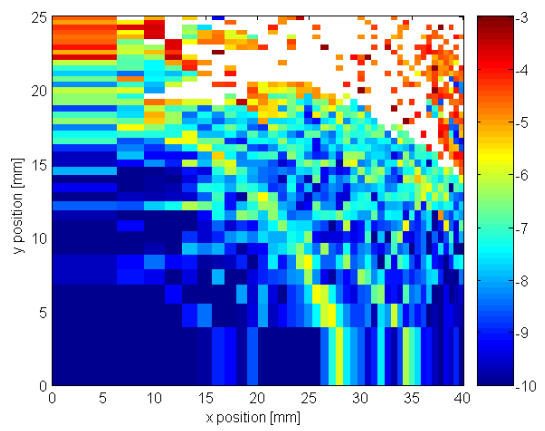

(b) $\xi_{x}=\xi_{y}=3$

Figure 11: Tune diffusion versus amplitude for the OCS6 lattice for different chromaticities. Resonances up to fifth order are shown.

nominal case ( $\xi_{x}=\xi_{y}=1$, see Fig. 6 on page 7 ). Also for negative momentum deviation it looks more frayed than the on-momentum case.

Figure 13 on page 13 shows the frequency maps for $\xi_{x}=\xi_{y}=3$. As in the nominal case, one can see that the horizontal third-integer resonance is strong, especially for negative momentum deviations, limiting the dynamic aperture significantly. One can see that the third-order resonance is responsible for the frayed shape of the footprints with negative momentum deviations, as in that case it is crossed at fairly low amplitude, causing loss of particles and high tune diffusion rates.

Figure 14 on page 14 shows the same data plotted versus initial amplitude. For positive momentum deviations, particles at large vertical amplitude are lost or have a high tune diffusion rate. For negative momentum deviations, this happens at even lower vertical amplitudes. The area with low tune diffusion rates is small, with the vertical quarter-integer as the first visible resonance.

For off-momentum particles, higher chromaticity than normal clearly decreases the dynamic aperture further and leads to large tune diffusion rates even for moderate amplitudes, especially in the vertical plane for negative momentum deviation.

\subsubsection{Chromaticity scan}

Figure 15 on page 15 shows a scan of both chromaticities for on-momentum particles. Here only the sum of all diffusion rates for a whole frequency map is 


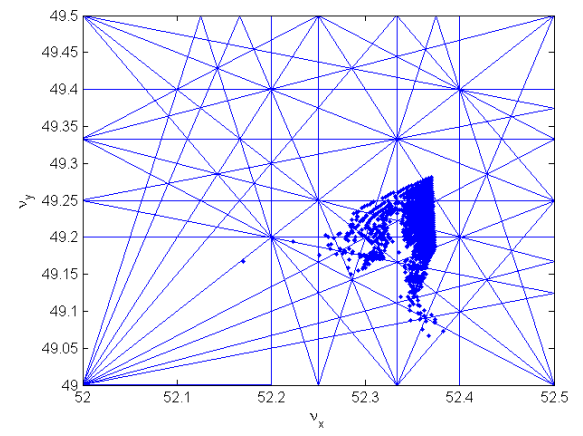

(a) $\frac{\Delta p}{p}=-0.5 \%$

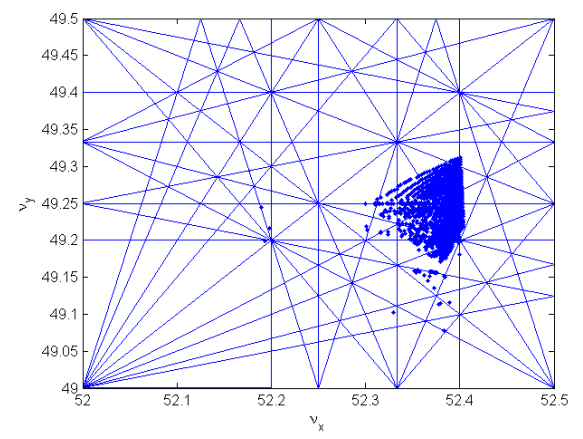

(c) $\frac{\Delta p}{p}=+0.5 \%$

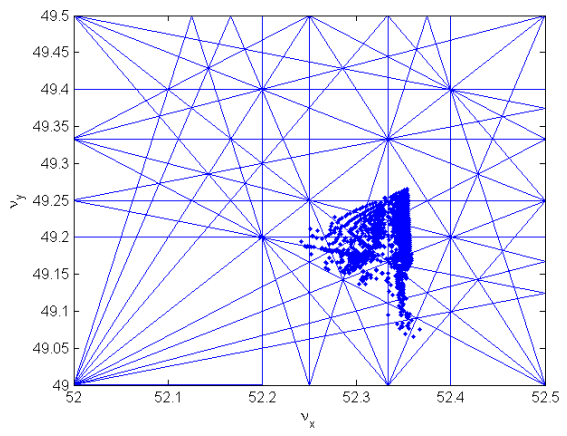

(b) $\frac{\Delta p}{p}=-1.0 \%$

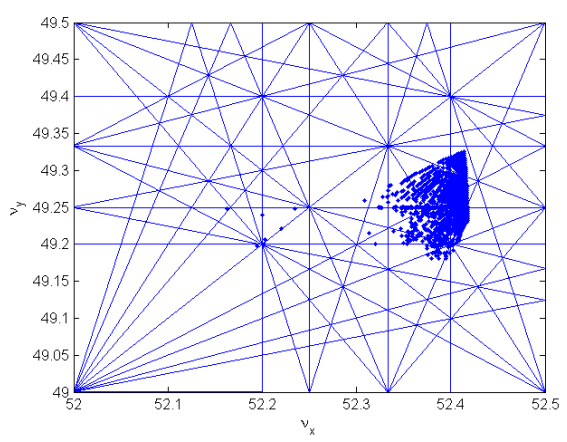

(d) $\frac{\Delta p}{p}=+1.0 \%$

Figure 12: Off-momentum footprints for the OCS6 lattice with $\xi_{x}=\xi_{y}=3$.

recorded. The horizontal chromaticity increases the diffusion rate whereas the vertical chromaticity lowers it very slightly. However, as for on-momentum, the influence of the sextupole strength on the trajectory is small, so this result is not very important.

The off-momentum results look very different. For negative momentum deviation (Fig. 16(a)) vertical chromaticity does not change the result much whereas increasing the horizontal chromaticity increases the diffusion rate and then, at very high values, lowers it again. This is due to the footprint being pushed across the horizontal third-order resonance (getting the tune close to the resonance increases the diffusion rate; after crossing the resonance, the diffusion rates are low again). For positive momentum deviation (Fig. 16(b)) increasing either chromaticity increases the diffusion rate. In some areas the change is stronger, possibly connected to crossing resonance lines. 


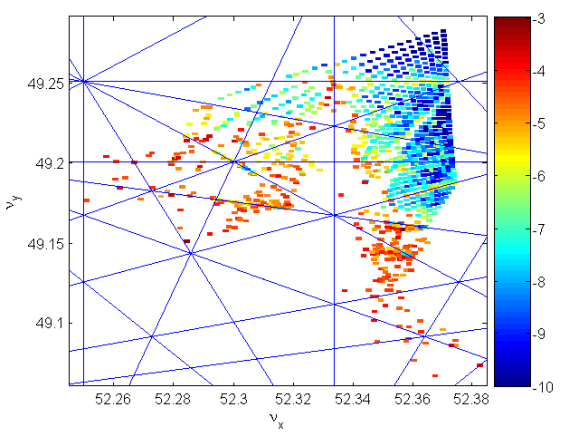

(a) $\frac{\Delta p}{p}=-0.5 \%$

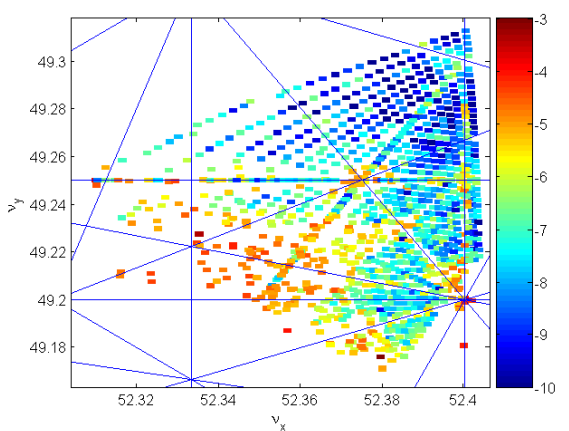

(c) $\frac{\Delta p}{p}=+0.5 \%$

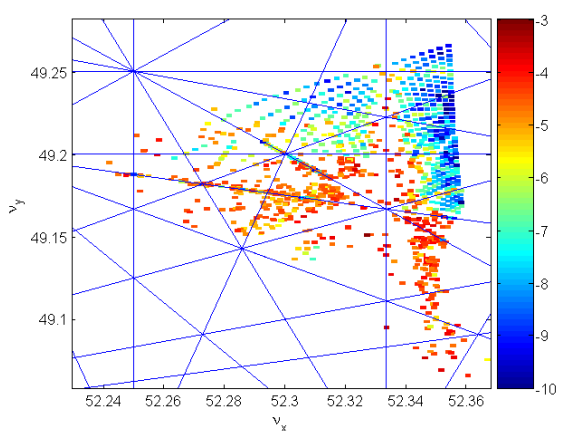

(b) $\frac{\Delta p}{p}=-1.0 \%$

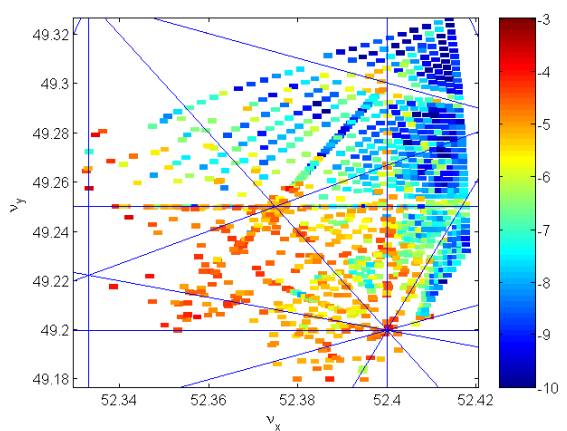

(d) $\frac{\Delta p}{p}=+1.0 \%$

Figure 13: Frequency maps for the OCS6 lattice with $\xi_{x}=\xi_{y}=3$.

Overall the chromaticity should be kept as small as possible to avoid particle losses. At least with the current lattice, already for $\xi_{x}=\xi_{y}=3$ the decrease in dynamic aperture is significant and higher chromaticities lead to even higher particle losses.

\section{Changing the tune of the ocs6 lattice}

\subsection{OCS6 with OCS5 tunes}

OCS5 has tunes of $Q_{x}=52.2810$ and $Q_{y}=47.4012$ compared to $Q_{x}=52.388$ and $Q_{y}=49.302$ for OCS6. It was suspected that the reduced dynamic aperture might be due to the different tune. Therefore the tune in OCS6 was adjusted to $Q_{x}=$ 52.2810 and $Q_{y}=49.4012$ and frequency maps were calculated to determine 


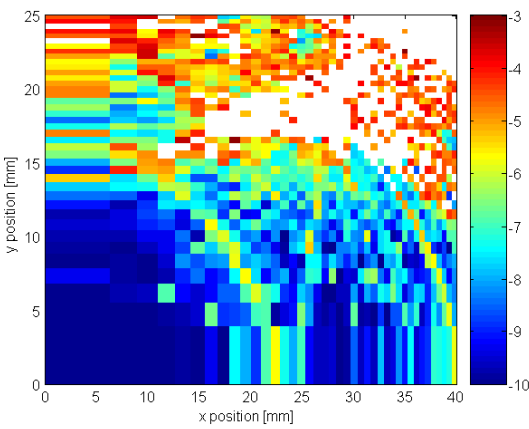

(a) $\frac{\Delta p}{p}=-0.5 \%$

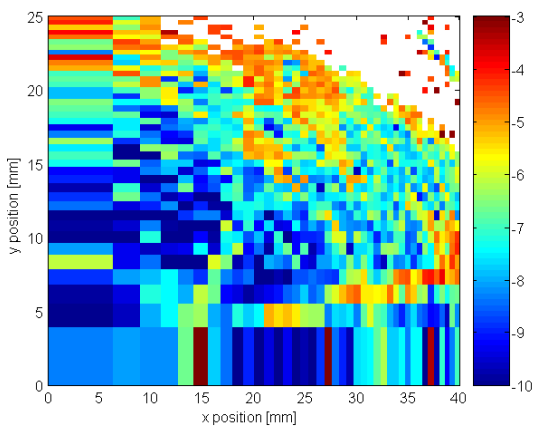

(c) $\frac{\Delta p}{p}=+0.5 \%$

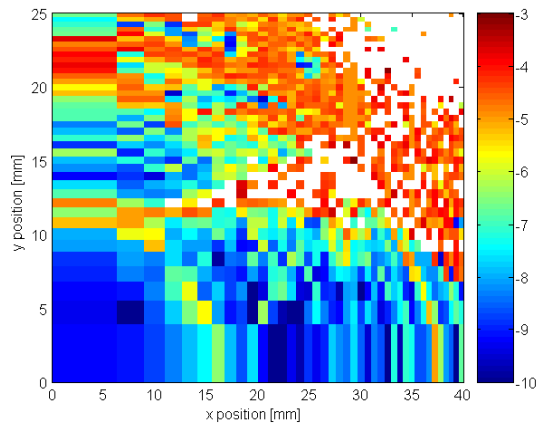

(b) $\frac{\Delta p}{p}=-1.0 \%$

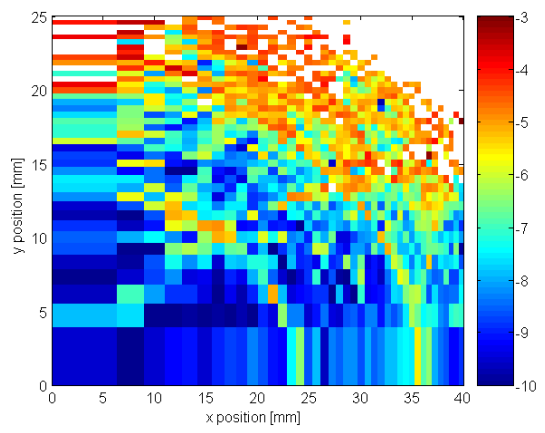

(d) $\frac{\Delta p}{p}=+1.0 \%$

Figure 14: Tune diffusion versus amplitude for the OCS6 lattice with $\xi_{x}=\xi_{y}=3$.

wether this is indeed the source of the reduced dynamic aperture. Two different methods of adjusting the tune were used:

1. Using one of the long straight sections (the so-called 'idle' straight).

2. Using the quadrupoles in the arc cells.

Both methods have their advantages and disadvantages which are discussed below. No optimization beyond keeping the chromaticities at $\xi_{x}=\xi_{y}=1$ was done.

\subsubsection{Using one long straight section as tune trombone}

The idle straight section consists mainly of a FODO structure with a chain of focusing and defocusing quadrupoles. At each end are four additional quadru- 


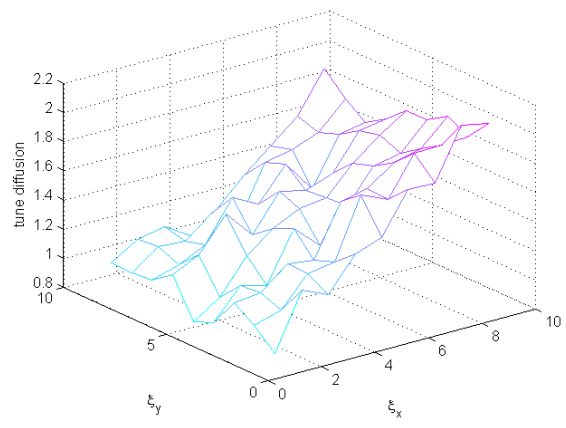

(a)

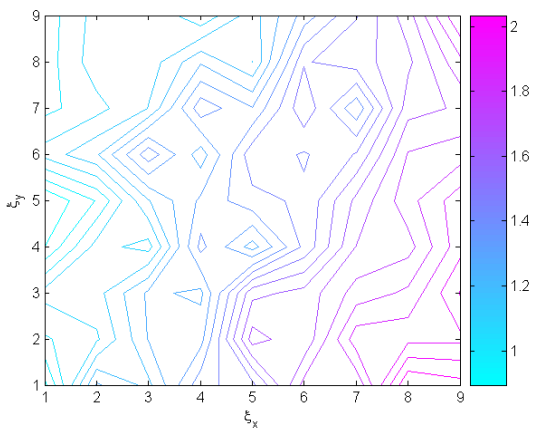

(b)

Figure 15: On-momentum tune diffusion for different chromaticities.

poles to match into the arc. All quadrupoles in the straight were used for the tune change. This method has the advantage of not changing the phase advance between sextupole magnets. On the other hand the whole tune change is lumped and it is not helpful if the source of the reduced dynamic aperture is the phase advance in the arcs. As the arc cells in OCS5 and OCS6 are identical, it is safe to assume that this is not the cause of the reduced dynamic aperture in the OCS6 lattice.

As the launch point for the tracking is located in the center of the 'idle' straight, the change in $\beta$-function at the launch point has to be taken into account. Therefore in this case the amplitudes are adjusted to correspond to the same normalized amplitudes as in all the other cases.

Figure 17(a) on page 17 shows the footprint with the adjusted tune. The footprint is significantly smaller but this is caused by particles losses, not by the detuning with amplitude being smaller.

Figure 18(a) on page 17 shows the frequency map for the adjusted tune space. It looks like problems are being caused by the horizontal fourth-integer and vertical third-integer resonances. It looks like the resonances are significantly stronger in the OCS6 lattice compared to the OCS5 lattice. They are visible in the frequency map for the OCS5 lattice (Fig. 4(b) on page 5) but there they are significantly weaker.

Figure 19(a) on page 18 shows the same data plotted versus initial amplitude instead of tune. Here the large losses and significantly reduced dynamic aperture are clearly visible. The horizontal fourth-order resonance causes significant particle losses even at moderate amplitudes. The dynamic aperture is 

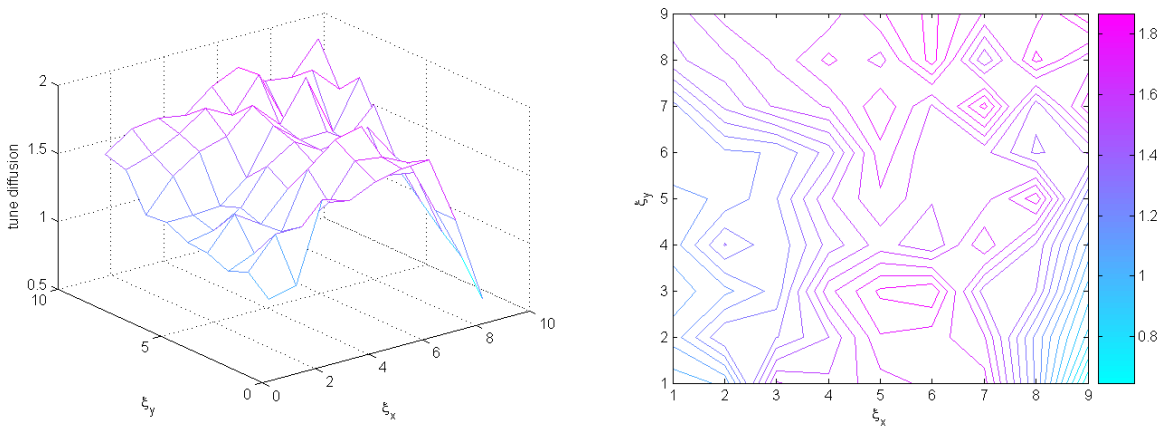

(a) $\frac{\Delta p}{p}=-0.5 \%$
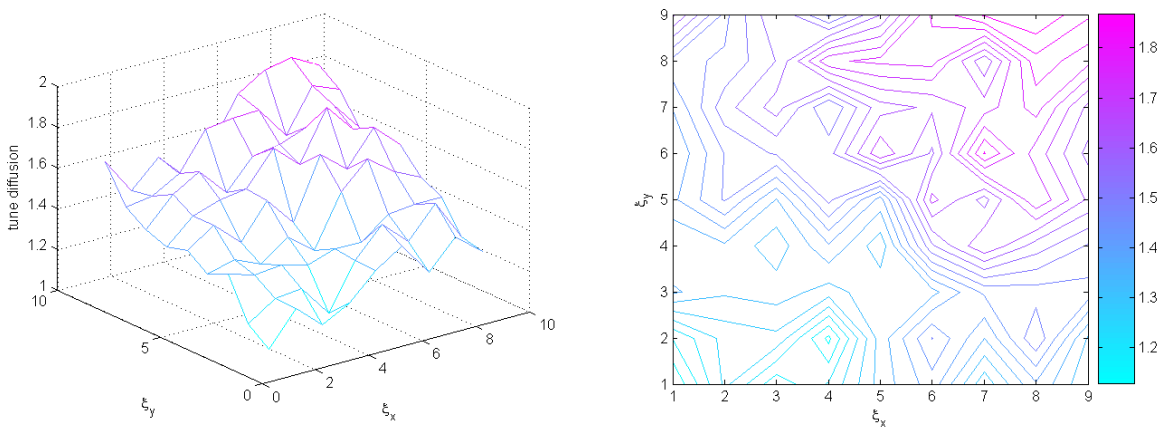

(b) $\frac{\Delta p}{p}=0.5 \%$

Figure 16: Off-momentum tune diffusion for different chromaticities.

a lot smaller than at the nominal tune, therefore suggesting that the tune itself might not be the source of the reduced dynamic aperture.

\subsubsection{Changing the phase advance in the arcs}

The arc has one chain of focusing and defocusing quadrupoles each. As the sextupole magnets used to correct the chromaticity are located in the arc as well, changing the tune with the arc quadrupoles changes the phase advance between the sextupoles. This can reduce the dynamic aperture which is the drawback of this method. The advantage is that the correct setting of the quadrupoles for the desired tune can easily be calculated and no matching is required. The change in quadrupole strength is rather small (nominal tune: $k_{\mathrm{QF}}=0.2853$ and $k_{\mathrm{QD}}=-0.2870$; old tune: $k_{\mathrm{QF}}=0.2849$ and $\left.k_{\mathrm{QD}}=-0.2872\right)$. The phase advance between two sextupoles of the same family changes from $\mu_{x}=\frac{\pi}{2}$ and 


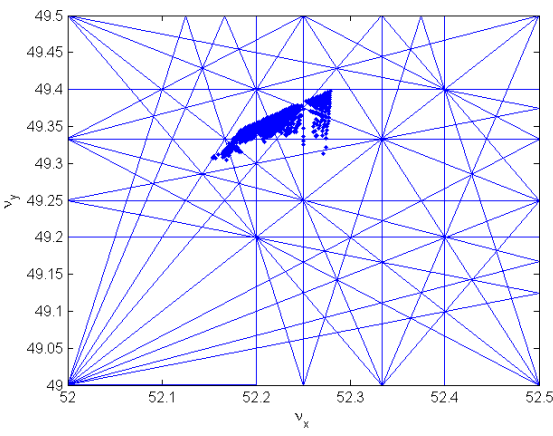

(a) straight section as tune trombone

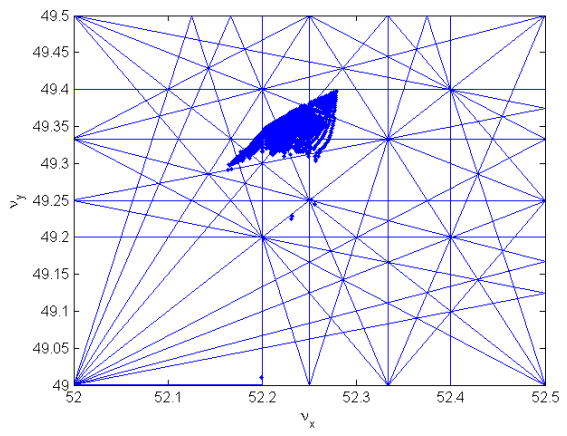

(b) changed phase advance in arc

Figure 17: Tune footprints for the OCS6 lattice for a tune of $Q_{x}=52.2810$ and $Q_{y}=$ 49.4012. Resonances up to fifth order are shown.

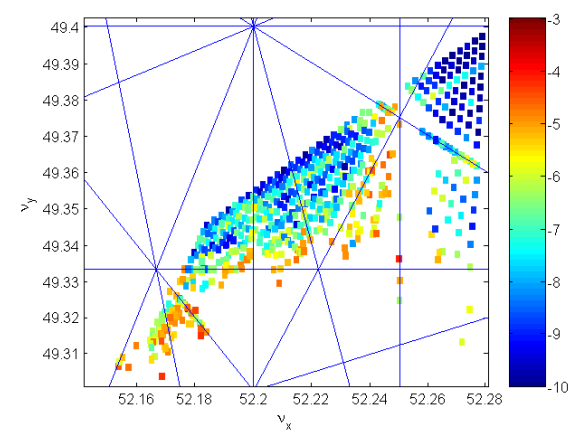

(a) straight section as tune trombone

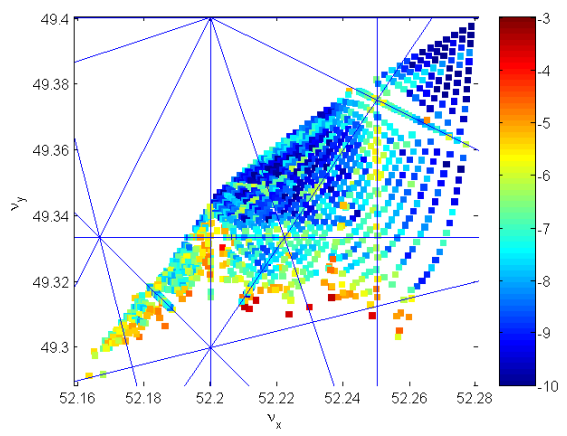

(b) changed phase advance in arc

Figure 18: Frequency maps for the OCS6 lattice for a tune of $Q_{x}=52.2810$ and $Q_{y}=$ 49.4012. Resonances up to fifth order are shown.

$\mu_{y}=\frac{\pi}{2}$ to $\mu_{x}=0.996 \times \frac{\pi}{2}$ and $\mu_{y}=1.003 \times \frac{\pi}{2}$. This change in phase advance is small enough that it is assumed to cause no significant reduction in dynamic aperture.

Figure 17(b) shows the footprint for the adjusted tune. The footprint looks very similar to the one of the OCS5 lattice (Fig. 3(b) on page 5). It is only slightly larger. It is not yet understood why the detuning with amplitude at this tune is significantly smaller than at the nominal tune for the Ocs6 lattice.

Figure 18(b) shows the frequency map for the adjusted tune. Again it looks comparable to the one of the OCS5 lattice (Fig. 4(b) on page 5) except that some 


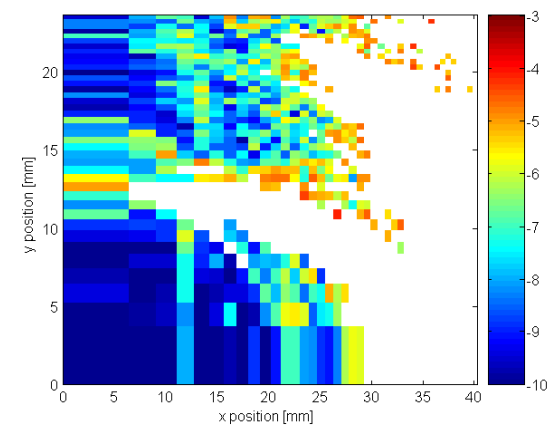

(a) straight section as tune trombone

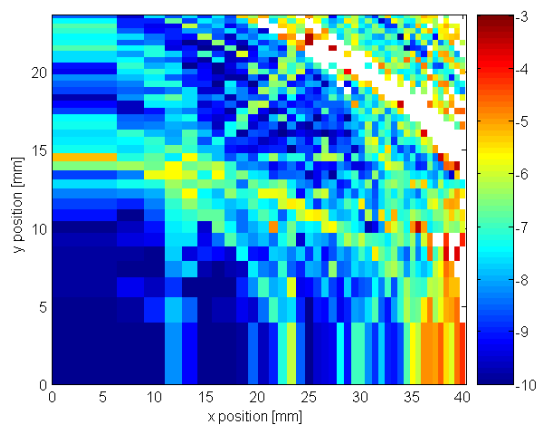

(b) changed phase advance in arc

Figure 19: Tune diffusion versus amplitude for the ocs6 lattice for a tune of $Q_{x}=$ 52.2810 and $Q_{y}=49.4012$. Resonances up to fifth order are shown. Amplitudes are adjusted to account for different $\beta$-functions at the launch point.

resonances are stronger (especially the horizontal fifth-order resonance) and the footprint is larger and frayed at the bottom.

Figure 19(b) shows the same data plotted versus initial amplitude. It shows that some particle losses at high amplitude occur mainly due to the horizontal fifth-order resonance. The resonances are stronger than the same ones in the OCS5 lattice and occur at lower amplitude due to the larger detuning with amplitude. From this, it looks like the tune is at least part of the dynamic aperture problem (with the larger detuning with amplitude being the other cause). The large detuning with amplitude could be decreased by using sextupole magnets in the straight sections (harmonic sextupoles). This has not yet been studied.

\subsection{Tune scan for the ocs6 lattice}

In order to explore if better areas in tune space exist, a tune scan using the quadrupole magnets in the arcs was done. As the change in quadrupole strength can be calculated analytically, no matching is required, making this approach faster than other methods of changing the tune. The only optimization done at each step was setting the chromaticities back to $\xi_{x}=\xi_{y}=1$. As for the chromaticity scan, only the sum of the diffusion rates is recorded. Results are plotted in Fig. 20. Both plots show the same data. The locations of the nominal tunes of OCS5 and OCS6 are marked in Fig. 20(b). Fig. 20(a) shows a mesh plot where each crossing of lines corresponds to a point for which the tune diffusion rate 
was actually calculated. Fig. 20(b) is a contour plot based on the same data which makes it easier to identify areas of low tune diffusion rates.

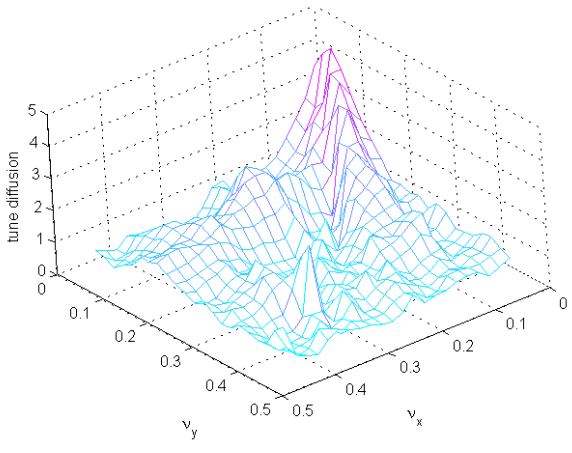

(a)

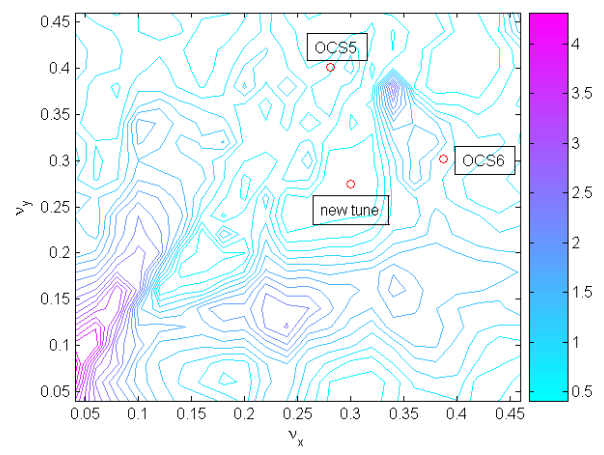

(b)

Figure 20: Tune diffusion rates for different tunes.

The lowest diffusion rate occurs in the region around $v_{x}=v_{y}=0.3$. As this area is close to the coupling resonance a tune was chosen for further study such that the footprint would not intersect the coupling resonance. The tune used for further study is $Q_{x}=52.300$ and $Q_{y}=49.275$. Figure 21 on the next page shows the tune footprint for the nominal tune (Fig. 21(a)) and the new tune (Fig. 21(b)). The footprint is significantly smaller, and this is not caused by particle losses. It looks like the cross detuning terms are smaller at this tune. The footprint stays below the coupling resonance, as intended.

Figure 22 on the following page shows the frequency map for the new tune location (Fig. 22(b)) and the one for the nominal case for comparison (Fig. 22(a)). Some resonances are visible but they are not very strong. The strongest one is the one below the vertical fifth-order resonance which corresponds to $v_{x}+$ $4 v_{y}=N$. The vertical fourth-order resonance causes only slightly increased diffusion rates.

Figure 23(b) on page 21 shows the diffusion rates for the new tunes plotted versus initial amplitude. Again the results from the nominal case are shown for comparison (Fig. 23(a)). One can see that with the new tunes, the dynamic aperture is significantly larger than for the nominal case. However some resonances are visible at fairly small amplitude, especially the vertical fourth order resonance at $A_{x}=20 \mathrm{~mm}$ and $A_{y}=13 \mathrm{~mm}$. Up to vertical amplitudes of around $A_{y}=15 \mathrm{~mm}$ the diffusion rates are low. However above that they are high in 


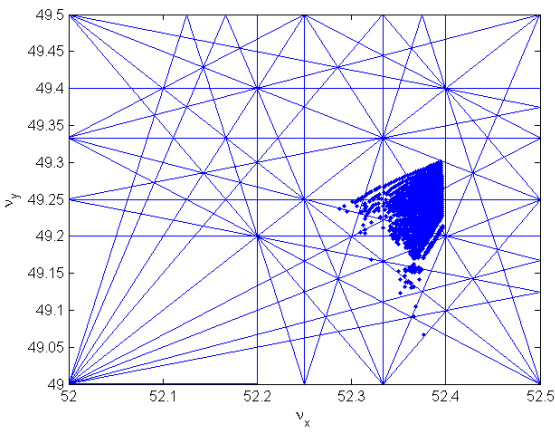

(a) $Q_{x}=52.388, Q_{y}=49.302$

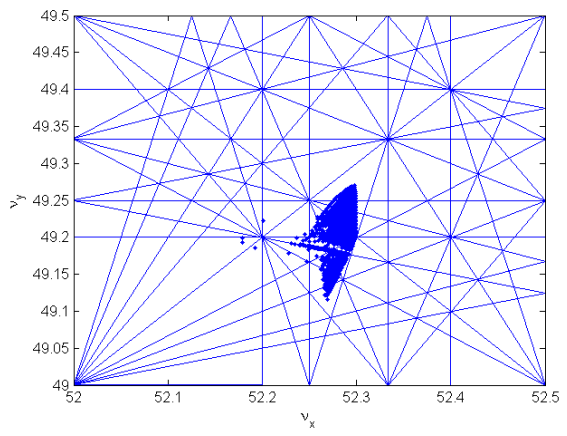

(b) $Q_{x}=52.300, Q_{y}=49.275$

Figure 21: Tune footprint for the OCS6 lattice for different tunes. Resonances up to fifth order are shown.

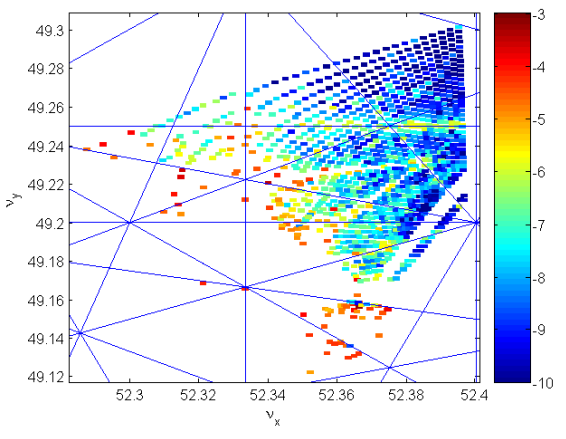

(a) $Q_{x}=52.388, Q_{y}=49.302$

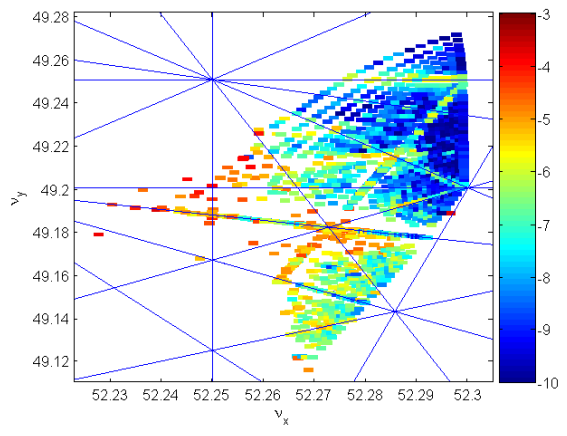

(b) $Q_{x}=52.300, Q_{y}=49.275$

Figure 22: Frequency maps for the OCS6 lattice for different tunes. Resonances up to fifth order are shown.

some areas.

Figure 24 on the following page shows the off-momentum footprints for the new tune values. The position changes slightly $\left(\xi_{x}=\xi_{y}=1\right)$ but the size and shape stay about the same.

Figure 25 on page 22 shows the off-momentum frequency maps for the new tune location. The upper part looks very good with the quarter integer resonance visible but not strong. The lower part shows large tune diffusion rates, not all of which can be attributed to particular resonance lines.

Figure 26 on page 22 shows the off-momentum tune diffusion rates plotted 


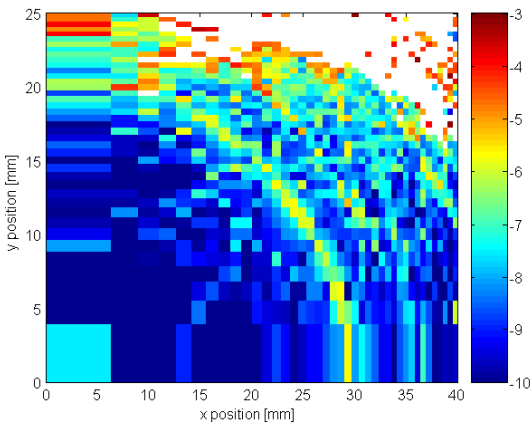

(a) $Q_{x}=52.388, Q_{y}=49.302$

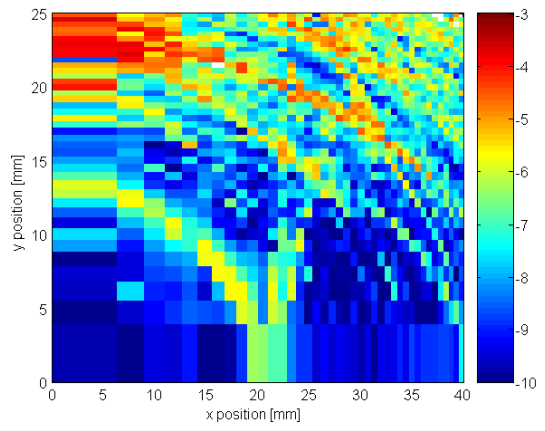

(b) $Q_{x}=52.300, Q_{y}=49.275$

Figure 23: Tune diffusion versus amplitude for the OCS6 lattice for different tunes. Resonances up to fifth order are shown.

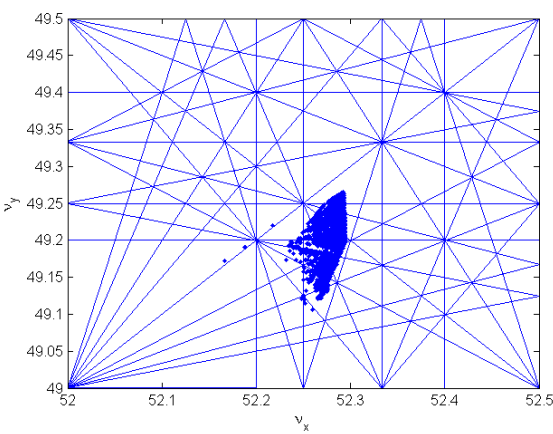

(a) $\Delta p / p=-0.5 \%$

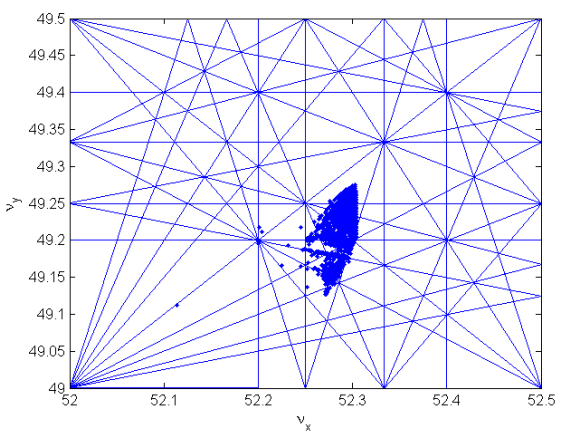

(b) $\Delta p / p=+0.5 \%$

Figure 24: Off-momentum tune footprint for the OCS6 lattice for $Q_{x}=52.300$ and $Q_{y}=$ 49.275. Resonances up to fifth order are shown.

versus initial amplitude. One can see that very few particles are lost compared to the nominal case (Fig. 8 on page 9). Large tune diffusion rates occur mainly at large vertical amplitudes and the vertical quarter-integer resonance is clearly visible at small amplitudes. For lower amplitudes the diffusion rates are low.

The dynamic properties at the new tune look significantly better than the nominal case for the OCS6 lattice, but are still worse than the OCS5 lattice mainly for large vertical amplitudes. However, one must keep in mind that no optimization was done beyond choosing a random tune in a good area from the tune scan (only making sure the footprint stays below the coupling resonance) 


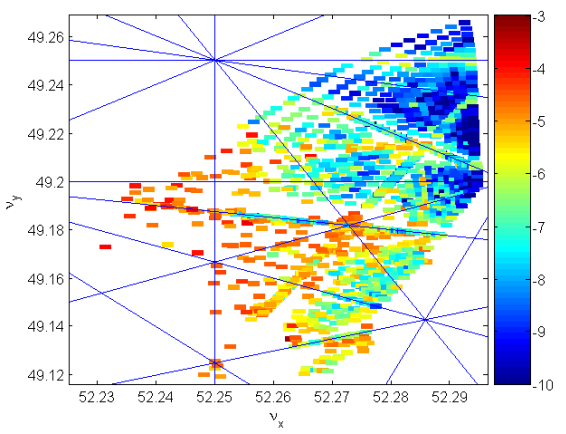

(a) $\Delta p / p=-0.5 \%$

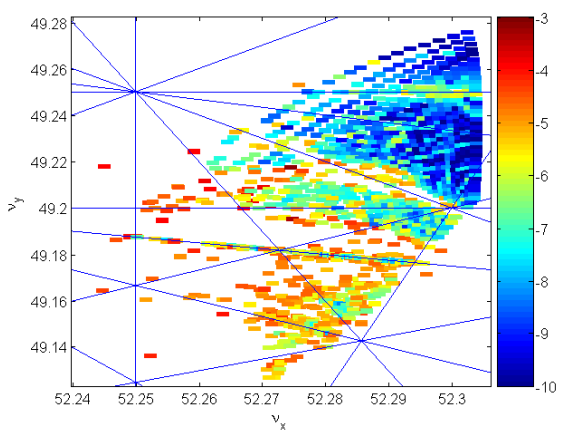

(b) $\Delta p / p=+0.5 \%$

Figure 25: Off-momentum frequency map for the ocs6 lattice for $Q_{x}=52.300$ and $Q_{y}=$ 49.275. Resonances up to fifth order are shown.

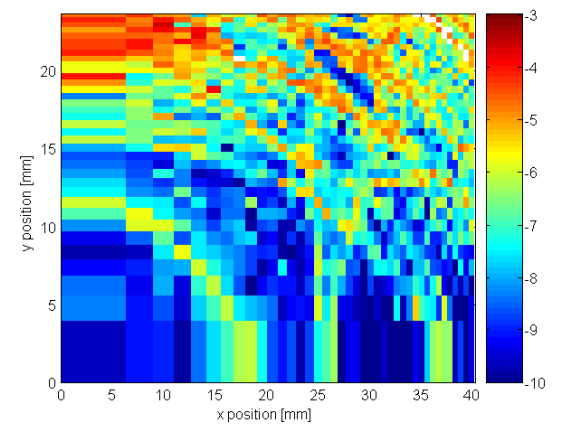

(a) $\Delta p / p=-0.5 \%$

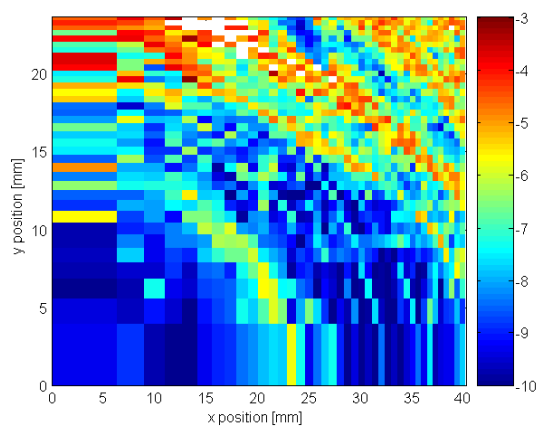

(b) $\Delta p / p=+0.5 \%$

Figure 26: Off-momentum diffusion rate versus amplitude for the OCS6 lattice for $Q_{x}=$ 52.300 and $Q_{y}=49.275$.

and keeping the chromaticities at $\xi_{x}=\xi_{y}=1$. So a more thorough study of that area might yield a good tune with a more optimized dynamic aperture. Harmonic sextupoles might improve the dynamic aperture even more by lowering the detuning with amplitude. This has not yet been studied.

\section{Conclusion}

The current version of the OCS6 lattice has a marginal dynamic aperture, even for a nominal chromaticity $\left(\xi_{x}=\xi_{y}=1\right)$. Larger chromaticities further decrease 
the dynamic aperture.

As the tune in older versions of the lattice, which had larger dynamic aperture, was different, frequency maps for different tunes were studied. Although the dynamic aperture at the location of the old tune is not better than for the nominal one, a different area in tune space looks promising. One tune in that area has been studied and yields a significantly larger dynamic aperture than the nominal case. Further studies in that area might yield a tune with a better dynamic aperture. Failing that harmonic sextupoles might be helpful to reduce the detuning with amplitude as this is one of the reasons of the reduced dynamic aperture.

All studies were done using no errors. Including machine errors like alignment, strength or multipole errors is likely to decrease the dynamic aperture. So studies of the final lattice should include the influence of those errors on the dynamic aperture.

\section{List of Figures}

1 Layout and Twiss parameters of the ocs6 lattice. . . . . . . . . . . 3

2 Layout and Twiss parameters of the OCS5 lattice. . . . . . . . . . . 4

3 Tune footprints for the OCS5 and ocs6 lattices. . . . . . . . . . 5

4 Frequency maps for the OCS5 and ocs6 lattices. . . . . . . . . . 5

5 Tune diffusion versus amplitude for the OCS5 and OCS6 lattices. . . 6

6 Off-momentum footprints for the ocs6 lattice. . . . . . . . . . 7

7 Off-momentum frequency maps for the ocs6 lattice. . . . . . . . . 8

8 Off-momentum tune diffusion versus amplitude for the ocs6 lattice. 9

9 Tune footprint for the OCS6 lattice for different chromaticities. . . 10

10 Frequency maps for the Ocs6 lattice for different chromaticities. . 10

11 Tune diffusion versus amplitude for the OCS6 lattice for different chromaticities. . . . . . . . . . . . . . . 11

12 Off-momentum footprints for the ocs6 lattice with $\xi_{x}=\xi_{y}=3 . \quad$. 12

13 Frequency maps for the ocs6 lattice with $\xi_{x}=\xi_{y}=3 . \ldots 13$

14 Tune diffusion versus amplitude for the OCS6 lattice with $\xi_{x}=\xi_{y}=3.14$

15 On-momentum tune diffusion for different chromaticities. . . . . 15

16 Off-momentum tune diffusion for different chromaticities. . . . . 16

17 Tune footprints for the OCS6 lattice for a tune of $Q_{x}=52.2810$ and

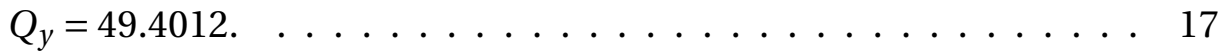


18 Frequency maps for the OCS6 lattice for a tune of $Q_{x}=52.2810$ and

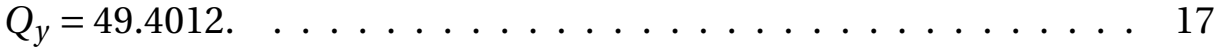

19 Tune diffusion versus amplitude for the ocs6 lattice for a tune of $Q_{x}=52.2810$ and $Q_{y}=49.4012 \ldots \ldots \ldots \ldots \ldots$

20 Tune diffusion rates for different tunes. . . . . . . . . . . . . 19

21 Tune footprint for the OCs6 lattice for different tunes. . . . . . . . . 20

22 Frequency maps for the OCS6 lattice for different tunes. . . . . . . 20

23 Tune diffusion versus amplitude for the OCS6 lattice for different

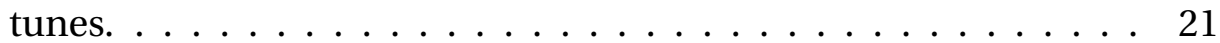

24 Off-momentum tune footprint for the ocs6 lattice for $Q_{x}=52.300$

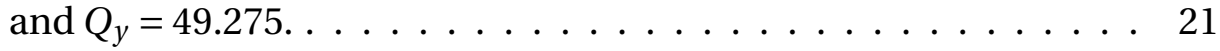

25 Off-momentum frequency map for the OCS6 lattice for $Q_{x}=52.300$

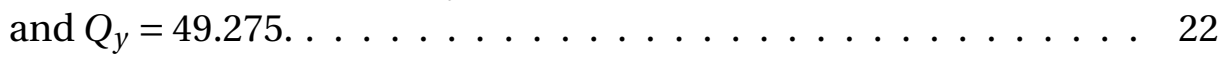

26 Off-momentum diffusion rate versus amplitude for the ocs6 lattice for $Q_{x}=52.300$ and $Q_{y}=49.275 \ldots \ldots \ldots \ldots \ldots \ldots \ldots$. . . . . . 22

\section{References}

[1] J. Laskar. Frequency Map Analysis and Particle Accelerators. In Proceedings of the 2003 Particle Accelerator Conference, 2003. 